\title{
Testing the critical exponent in the relation between stress drop of earthquake and lead time of seismic electric signal
}

\author{
E. Dologlou \\ Solid State Section, Department of Physics, University of Athens, Panepistimiopolis, Zografou 15784 Athens, Greece \\ Correspondence to: E. Dologlou (edologl@phys.uoa.gr)
}

Received: 6 June 2012 - Accepted: 26 June 2012 - Published: 16 August 2012

\begin{abstract}
The application of new data in the power law relation between the stress drop of the earthquake and the lead time of the precursory seismic electric signal led to an exponent which falls in the range of the values of critical exponents for fracture and it is in excellent agreement with a previous one found by (Dologlou, 2012). In addition, this exponent is very close to the one reported by Varotsos and Alexopoulos (1984a), which interconnects the amplitude of the precursory seismic electric signals (SES) and the magnitude of the impending earthquake. Hence, the hypothesis that underlying dynamic processes evolving to criticality prevail in the pre-focal area when the SES is emitted is significantly supported.
\end{abstract}

\section{Introduction}

The preparation process of earthquakes is treated under the light of the new aspects of the critical point earthquake model, which predicts that failure is a co-operative effect occurring at small scale and cascading from the microscopic to the macroscopic scale (Allègre and Le Mouel, 1994; KeilisBorok, 1990). The concept that earthquakes can be viewed as critical phenomena is supported by recent observations that rupture in heterogeneous media exhibits critical behavior (Andersen et al., 1997; Lamaignere et al., 1996; Sornette, 2000). The word "critical" describes a system at the boundary between order and disorder, and is characterized by both extreme susceptibility to external factors and strong long range correlations between different parts of the system (Sornette and Sornette, 1990). During the last preparatory stage in the pre-focal region, power law procedures (characteristic of criticality) control the evolution of earthquake dynamic processes (Bowman et al., 1998; Teotia and Kumar, 2011), which end up by culminating in the cataclysm event of the main shock.

In a series of recent articles a power law relation between dynamic parameters of earthquakes and associated precursory seismic electric signals (SES) based on progressively updated data has been reported (Dologlou, 2008, 2009, 2010, 2012). Seismic electric signals (SES) are low frequency $(<1 \mathrm{~Hz})$ transient signals embedded on the earth's telluric field. They are emitted from the pre-focal area when the tectonic stress reaches a critical value which also signals the entrance of the region into the last earthquake preparatory phase. SES are continuously monitored the last three decades by a network covering continental Greece and have been found to precede large earthquakes (Varotsos and Alexopoulos, 1984a, b; Varotsos et al., 1986, 1988, 1993, 2009). A possible model for SES generation which is based on the existence of point defects in solids (Varotsos and Alexopoulos, 1977, 1981; Varotsos and Ludwig, 1978; Varotsos et al., 1978; Varotsos, 2007a, b, 2008) is as follows (e.g. see Varotsos et al., 1993 and references therein): rocks contain various materials with lattice defects for charge compensation when are doped with aliovalent impurities. The relaxation time of the electric dipoles which are formed between a portion of these defects and the impurities, decrease upon increasing pressure, provided that the migration volume is negative. When the pressure (tectonic stress) reaches a critical value a cooperative change of the dipole's orientation results in a transient current which constitutes the SES. A series of successive such signals forms a SES activity. It has been found (Varotsos et al., 2002, 2003a, b, 2007, 2008; Abe et al., 2005; Sarlis et al., 2010) that the SES activities exhibit scale invariant structure (power law) which is consistent with the 
Table 1. The USGS reported date and magnitude of the earthquake along with its seismic moment $M_{o}$, range of values in the dimensions of its aftershock area $\mathrm{L}$ and $\mathrm{W}$, corresponding calculated values for $\Delta \sigma_{B}$, the critical exponent $\alpha$ and the lead time $\Delta t$. Mean values are also given for the relevant parameters.

\begin{tabular}{lllllllll}
\hline $\mathrm{n}_{O}$ & Date & $M_{\mathrm{W}}$ & $M_{O}$ & $\mathrm{~L}$ & $\mathrm{~W}$ & $\Delta \sigma_{B}$ & $\alpha$ & $\Delta t$ \\
\hline \multirow{3}{*}{1} & yy mm dd & & $\left(10^{24} \mathrm{dyn} \mathrm{cm}\right)$ & $(\mathrm{km})$ & $(\mathrm{km})$ & $($ bars $)$ & & days \\
& 120416 & 5.8 & 5.6 & $24-26$ & $15-17$ & $1.48-2.00$ & $0.334-0.322$ & 101 \\
& Mean value & & & 25 & 16 & 1.71 & $0.329 \pm 0.01$ & \\
\hline
\end{tabular}

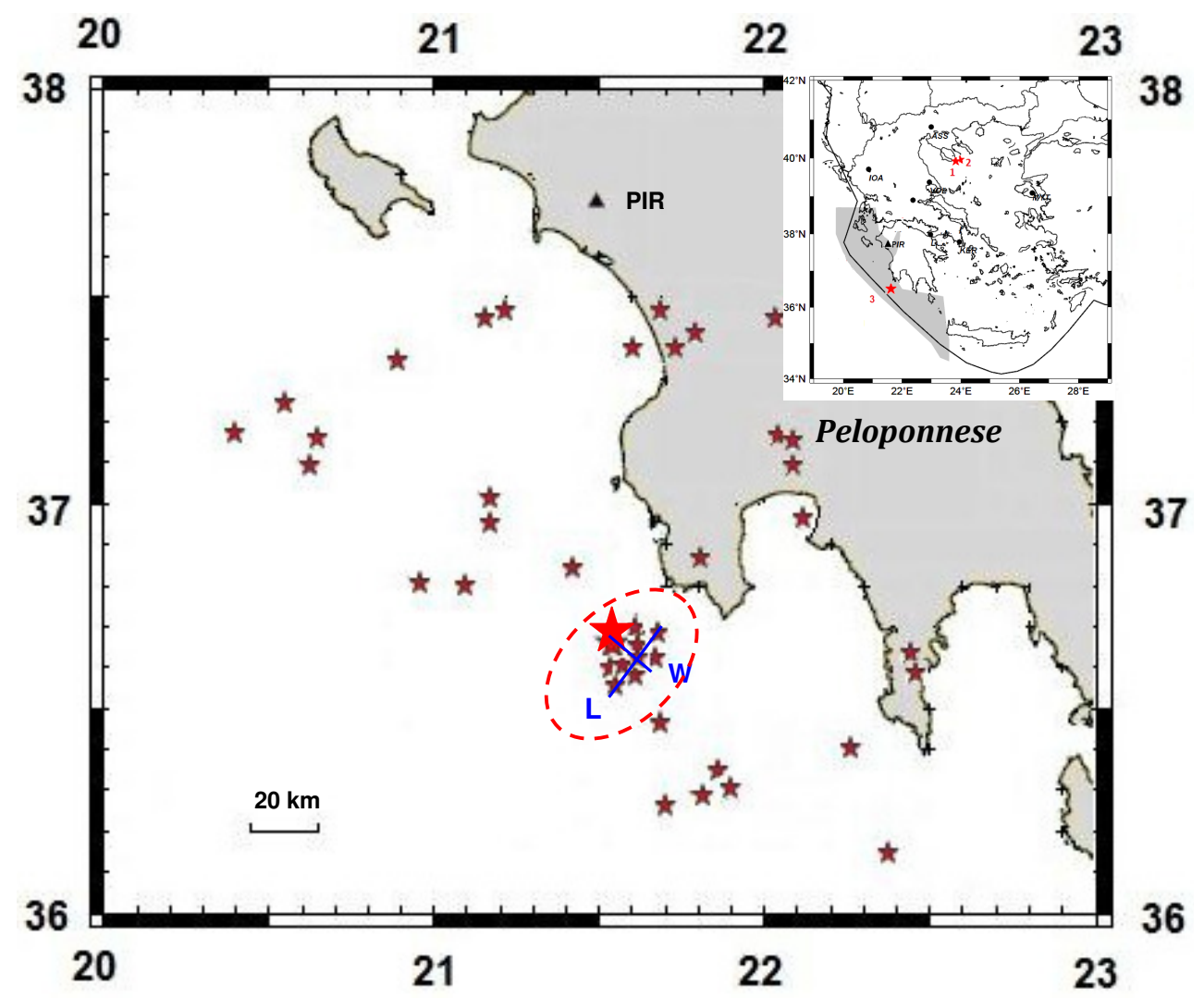

Fig. 1. Map of SW Greece, with all earthquakes (small red stars) with $M_{L} \geq 2.3$ reported by NOA from 16 April 2012 to 23 May 2012 in the area $(36-38)^{\circ} \mathrm{N},(20-23)^{\circ} \mathrm{E}$. A big red star denotes the epicenter of the $M_{\mathrm{W}}=5.8$ main shock on 16 April 2012, while a red dashed ellipse shows the location of the cluster of aftershocks. The cluster dimensions $W$ and $L$ are given in blue. A triangle depicts the location of the SES station PIR. The inset on the right top of the figure shows the selectivity map of PIR as shaded area, and the epicenters of the three earthquakes as red stars, numbered according to their order in the text. The $M_{\mathrm{W}}=5.8$ earthquake is the number 3 event.

criticality concept of the SES generation model mentioned above. The following experimental power law relation, characteristic of criticality, between the SES amplitude $E$ and the magnitude $M$ of the impending earthquake, has been found (Varotsos and Alexopoulos, 1984a see p. 91).

$\log E=\mathrm{a} M+\mathrm{b}$

where $E=\Delta V / L$ with $\Delta V$ the potential difference between to points on the ground at a distance $L, \mathrm{a} \approx 0.3-0.4$ and $\mathrm{b}$ is a site constant depending on the geoelectrical structure around the station. Another characteristic of the SES is the selectivity effect which states that a SES station can be sensitive to specific seismic regions while remains inactive for others even at shorter distances (Varotsos and Lazaridou, 1991; Varotsos et al., 1996).

Here, by using recent new data, we test whether the very sensitive exponent of a power law relation previously obtained (Dologlou, 2009, 2010, 2012) between the stress drop of the earthquake (stress state difference at a point on a fault before and after the occurrence of the earthquake) and the lead time (time lag between SES emission and earthquake occurrence) of the precursory SES remains stable and within the range of critical values. 


\section{Data analysis and discussion}

In this study we deal with the next most recent data set (after those published by Dologlou, 2012) and we refer to the earthquakes with $M \geq 5$ that occurred in Greece and the corresponding available SES during the time period 1 November 2011 to 23 May 2012. During this period and in the area $(36-41)^{\circ} \mathrm{N}(19-25)^{\circ} \mathrm{E}$, only one SES activity has been reported (Skordas et al., 2012) on 6 January 2012 at PIR station situated in Peloponnese, SW Greece (Fig. 1), and three earthquakes with $M \geq 5$ occurred according to USGS. The first two events took place on 14 February 2012 and 4 March 2012, respectively, with same magnitude $M_{\mathrm{w}}=5.2$ and almost the same location $\left(40.13^{\circ} \mathrm{N}, 24.09^{\circ} \mathrm{E}\right)$ in $\mathrm{NE}$ Greece (red stars numbered 1 and 2 in the inset on the right top of Fig. 1). The third event which is the largest one with $M_{\mathrm{w}}=5.8$, (red star number 3 in the inset) occurred on 16 April 2012 in SW Greece off the Peloponnese coast with epicenter $\left(36.63^{\circ} \mathrm{N}, 21.48^{\circ} \mathrm{E}\right)$ and is the only one which falls inside the selectivity map of PIR station (shaded area in the inset of Fig. 1 see Sarlis et al., 2008), and thus corresponds to the above SES activity with a lead time $\Delta t=101$ days (Table 1). The epicenters of the other two events (number 1 and 2) lie in a totally different seismotectonic region in N Greece.

Based on a mounting number of available data during a long period from 1981 to 2011, the following power law relation between the earthquake Brune's stress drop $\Delta \sigma_{\mathrm{B}}$ and the associated SES lead time $\Delta t$ has been reported:

$\Delta \sigma_{\mathrm{B}}=8.17 \Delta t^{-\alpha}$

with exponent $\alpha=0.327$ (see Table 1 in Dologlou (2010) and comments therein and Dologlou (2012)). This relation is under a continuous updating process by the author. Here, by introducing a new data set, we will check the validity of the above relation and the credibility of the exponent $\alpha$. In order to calculate the Brune's stress drop $\Delta \sigma_{\mathrm{B}}$, we used the Hanks and Wyss (1972) relation:

$\Delta \sigma_{B}=0.44 M_{o} / r^{3}$

with $M_{o}$ the seismic moment of the earthquake and $r$ the radius for a circular fault. The radius $r$ can be estimated from the aftershock area according to a technique proposed by Kiratzi et al. (1991). For this purpose, and considering as main shock the third event (number 3) with $M_{\mathrm{w}}=5.8$, we plotted on a map the epicenters of all earthquakes with $M_{L} \geq 2.3$ (Fig. 1 red stars) in the area $(36-38)^{\circ} \mathrm{N},(20-23)^{\circ} \mathrm{E}$ from 16 April 2012 to 23 May 2012, which are reported by the National Observatory of Athens (NOA) http://www.gein.noa.gr. A cluster is formed (denoted by a dashed ellipse in Fig. 1) around the main shock (big red star Fig. 1), defining the aftershock area of surface $S=L \times W$ (Fig. 1) with length $L=25 \mathrm{~km}$ and width $W=16 \mathrm{~km}$ (Table 1 ). The radius $r$ is derived from the relation $S=\pi r^{2}$. The seismic moment for the main shock $M_{o}=5.6 \times 10^{24} \mathrm{dyn} \mathrm{cm}$ (Table 1 ) is computed from the relation $\log \left(M_{o}\right)=1.5\left(M_{\mathrm{W}}+10.7\right)$ of Hanks

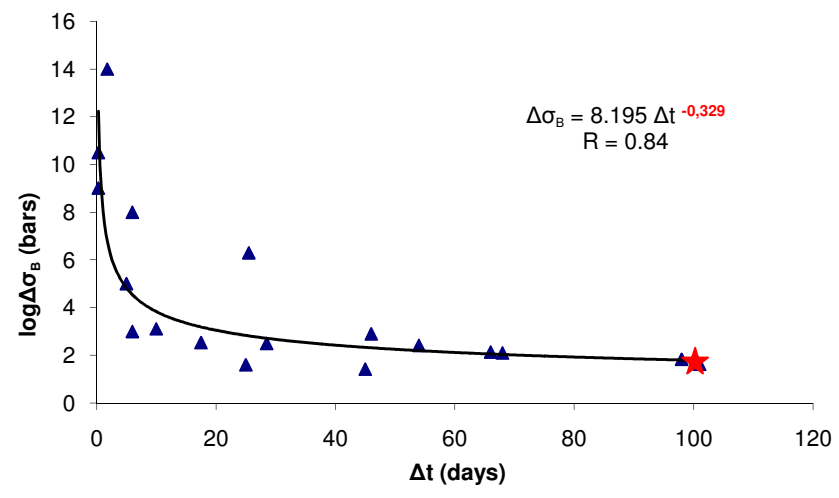

Fig. 2. The plot of the power law between the stress drop of the earthquakes and the lead time of SES. A red star corresponds to the new data (Table 1) while solid triangles refer to the data sets reported by Dologlou $(2010,2012)$. The derived power law relation along with its correlation coefficient $R$ and the exponent $\alpha=0.329$ are displayed on the top of the diagram.

and Kanamori (1979). Through Eq. (3) we calculate the Brune's stress drop $\Delta \sigma_{\mathrm{B}}=1.71$ bars (Table 1). By introducing in Eq. (2) the data set $\Delta \sigma_{\mathrm{B}}=1.71$ bars and $\Delta t=101$ days we get the value of the exponent $\alpha=0.329$ (see Table 1 and Fig. 2). This value is almost the same with the previous one 0.327 found by Dologlou (2012) and is in excellent agreement with the power law exponent which interconnects the amplitude of the SES and the magnitude of the impending earthquake (Varotsos and Alexopoulos, 1984a see p. 91 and here Eq. 1). This confirms the credibility of the power law relation between the stress drop and the SES lead time and probably implies that underlying dynamic processes of mechanical and electromagnetic origin evolving to criticality prevail in the pre-focal area when the SES is emitted.

\section{Conclusions}

The application of new data (November 2011 to May 2012) in the power law relation between the stress drop of the earthquake and the lead time of the precursory SES led to an exponent $\alpha=0.329$ that is in excellent agreement with previous ones found in a series of articles by the author and confirms its credibility. On the other hand, this exponent falls in the range of the values of critical exponents suggested by various models for fracture and is very close to the reported one by Varotsos and Alexopoulos (1984a), which interconnects the amplitude of the SES and the magnitude of the impending earthquake. Hence, the hypothesis that underlying dynamic processes of mechanical and electromagnetic origin evolving to criticality are present in the pre-focal area when the SES is emitted is considerably supported. In other words, the basic concept (i.e., the approach to a critical stage) on which the SES generation and emission was originally proposed (Varotsos and Alexopoulos, 1984a, b) is strengthened by the new data presented in this paper. 
Edited by: M. E. Contadakis

Reviewed by: two anonymous referees

\section{References}

Abe, S., Sarlis, N. V., Skordas, E. S., Tanaka, H. K., and Varotsos, P. A.: Origin of the usefulness of the natural time representation of complex time series, Phys. Rev. Lett., 94, 170601, 4, doi:10.1103/PhysRevLett.94.170601, 2005.

Allègre, C. J. and Le Mouel, J. L.: Introduction of scaling techniques in brittle failure of rocks, Phys. Earth Planet Inter., 87, 85-93, 1994.

Andersen, J. V., Sornette, D. and Leung, K.-T.: Tri-critical behavior in rupture induced by disorder, Phys. Rev. Lett. 78, 2140-2143, 1997.

Bowman, D., Ouillon, G., Sammis, C. G., Sornette, A., and Sornette, D.: An Observational Test of the Critical Earthquake Concept, J. Geophys. Res., 103, 24359-24372, 1998.

Dologlou, E.: Power law relationship between parameters of earthquakes and precursory electrical phenomena, Nat. Hazards Earth Syst. Sci., 8, 977-983, doi:10.5194/nhess-8-977-2008, 2008.

Dologlou, E.: Power law relationship between parameters of earthquakes and precursory electrical phenomena revisited, Nat. Hazards Earth Syst. Sci., 9, 17-23, doi:10.5194/nhess-9-17-2009, 2009.

Dologlou, E.: Power law relationship between parameters of earthquakes and precursory electrical phenomena revisited II, Nat. Hazards Earth Syst. Sci., 10, 1403-1409, doi:10.5194/nhess-101403-2010, 2010.

Dologlou, E.: Stability of a power law relation between characteristics of earthquakes and electric precursors, Nat. Hazards Earth Syst. Sci., 12, 1783-1787, doi:10.5194/nhess-12-17832012, 2012.

Hanks, T. and Wyss, M.: The use of body wave spectra in the determination of seismic source parameters, Bull. Seism. Soc. Am., 62, 561-589, 1972.

Hanks, T. and Kanamori, H.: Moment magnitude scale, Geophys. Res., 84, 2348-2350, doi:10.1029/JB084iB05p02348, 1979.

Keilis-Borok, V.: The lithosphere of the Earth as a large nonlinear system, in Quo Vadimus: Geophysics for the Next Generation, Geophys. Monogr. Ser., 60, edited by: Garland, G. D. and Apel, J. R., 81-84, AGU, Washington DC, 1990.

Kiratzi, A. A., Wagner, G. S., and Langston, C. A.: Source Parameters of Some Large Earthquakes in Nortern Aegean Determined by Body Waveform Inversion, Pure Appl. Geophys. (PAGEOPH), 135, 515-527, 1991.

Lamaignere, L., Carmona, F., and Sornette, D.: Experimental realization of critical thermal fuses rupture, Phys. Rev. Lett., 77, 2738-2741, 1996.

Sarlis, N. V., Skordas, E. S., Lazaridou, M. S., and Varotsos, P. A.: Investigation of the seismicity after the initiation of a Seismic Electric Signal activity until the main shock, Proceedings of the Japan Academy, Ser. B, 84, 331-343, 2008.

Sarlis, N. V., Skordas, E. S., and Varotsos, P. A.: Nonextensivity and natural time: The case of seismicity, Phys. Rev. E, 82, 021110 , doi:10.1103/PhysRevE.82.021110, 2010.

Skordas, E., Sarlis, N., and Varotsos, P.: Effect of significant data loss on identifying electric signals that precede rupture by detrended fluctuation analysis in natural time, arXiv:cond.mat:1003.1383v10, 26 January, 2012.

Sornette, D.: Critical Phenomena in Natural Sciences, Springer, 2000.

Sornette, A. and Sornette, D.: Earthquake rupture as a critical point : Consequences for telluric precursors, Tectonophysics, 179, 327334, 1990.

Teotia, S. S. and Kumar, D.: Role of multifractal analysis in understanding the preparation zone for large size earthquake in the North-Western Himalaya region, Nonlin. Proc. Geophys., 18, 111-118, doi:10.5194/npg-18-111-2011, 2011.

Varotsos, P.: Calculation of point defect parameters in diamond, Phys. Rev. B, 75, 172107, p. 3, doi:10.1103/PhysRevB.75.172107, 2007a.

Varotsos, P.: Comparison of models that interconnect point defect parameters in solids with bulk properties, J. Appl. Phys., 101, 123503, 8 pp., http://dx.doi.org/10.1063/1.2745359, 2007b.

Varotsos, P.: Point defect parameters in b-PbF2 revisited, Solid State Ionics, 179, 438-441, 2008.

Varotsos, P. and Alexopoulos, K.: Calculation of the formation entropy of vacancies due to anharmonic effects, Phys. Rev. B, 15, 4111-4114, 1977.

Varotsos, P. and Alexopoulos, K.: Calculation of diffusion coefficients at any temperature and pressure from a single measurement. II, Heterodiffusion, Phys. Rev. B, 24, 3606-3609, 1981.

Varotsos, P. and Alexopoulos, K. : Physical properties of the variations of the electric field of the earth preceding earthquakes, I, Tectonophysics, 110, 73-98, 1984a.

Varotsos, P. and Alexopoulos, K.: Physical properties of the variations of the electric field of the earth preceding earthquakes, II. Determination of epicentre and magnitude, Tectonophysics, 110, 99-125, $1984 b$.

Varotsos, P. and Lazaridou, M.: Latest aspects of earthquake prediction in Greece based on seismic electric signals. I, Tectonophysics , 188, 321-347, 1991.

Varotsos, P. and Ludvig, W.: On a new analysis of the diffusion experiments under pressure, J. Phys. C. Solid. State, 11, L305L309, 1978.

Varotsos, P., Ludwig, W., and Alexopoulos, K.: Calculation of the formation volume of vacancies in solids, Phys. Rev. B, 18, 26832691, 1978.

Varotsos, P., Alexopoulos, K., and Lazaridou, M.: Latest aspects of earthquake prediction in Greece based on Seismic Electric Signals II, Tectonophysics, 224, 1-37, 1993.

Varotsos, P. A., Sarlis, N. V., and Skordas, E. S.: Long-range correlations in the electric signals that precede rupture, Phys. Rev. E, 66, 011902, doi:10.1103/PhysRevE.66.011902, 2002.

Varotsos, P. A., Sarlis, N. V., and Skordas, E. S.: Longrange correlations in the electric signals that precede rupture: Further investigations, Phys. Rev. E, 67, 021109, doi:10.1103/PhysRevE.67.021109, 2003a.

Varotsos, P. A., Sarlis, N. V., and Skordas, E. S.: Attempt to distinguish electric signals of a dichotomous nature, Phys. Rev. E., 68, 031106, doi:10.1103/PhysRevE.68.031106, 2003b.

Varotsos, P. A., Sarlis, N. V., and Skordas, E. S.: Detrended fluctuation analysis of the magnetic and electric field variations that precede rupture Chaos 19, 0231147, http://dx.doi.org/10.1063/1. 3130931, 2009. 
Varotsos, P. A., Nomicos, K., and Lazaridou, M.: Earthquake prediction and electric signals, Nature, 322, 120, 1986.

Varotsos, P. A., Nomicos, K., and Lazaridou, M.: Official earthquake prediction procedure in Greece, Tectonophysics, 152, 193-196, 1988.

Varotsos, P., Eftaxias, K., Lazaridou, M., Antonopoulos, G., Makris, J., and Poliyiannakis, J.: "Summary of the five principles suggested by P. Varotsos et al. (1996) and the additional questions raised in this debate", Geophys. Res. Lett., 23, 11, 1449-1452, 1996.
Varotsos, P. A., Sarlis, N. V., Skordas, E. S., and Lazaridou, M. S.: Identifying sudden cardiac death risk and specifying its occurrence time by analyzing electrocardiograms in natural time, Appl. Phys. Lett., 91, 064106, doi:10.1063/1.2768928, 3 pp., 2007.

Varotsos, P. A., Sarlis, N. V., Skordas, E. S., and Lazaridou, M. S.: Fluctuations, under time reversal, of the natural time and the entropy distinguish similar looking electric signals of different dynamics, J. Appl. Phys., 103, 014906, doi:10.1063/1.2827363, 2008. 